\title{
Corrections to the scaling of the second-order structure function in isotropic turbulence
}

\author{
Le Fang ${ }^{\text {a }}$ Wouter J.T. Bos ${ }^{\text {a }}$ Xiaozhou Zhou ${ }^{\mathrm{b}}$ Liang Shao ${ }^{\text {a }}$ Jean-Pierre Bertoglio ${ }^{\text {a }}$ \\ ${ }^{a}$ Laboratoire de Mécanique des Fluides et d'Acoustique, Université de Lyon École centrale de Lyon, 69134 Ecully, France \\ ${ }^{\mathrm{b}}$ Department of Mechanical Engineering, Center for Biomedical Engineering Research, University of Delaware, 126 \\ Spencer Laboratory, Newark, DE 19716, USA
}

\begin{abstract}
The approach of Obukhov assuming a constant skewness was used to obtain analytical corrections to the scaling of the second order structure function, starting from Kolmogorov's 4/5 law. These corrections can be used in model applications in which explicit expressions, rather than numerical solutions are needed. The comparison with an interpolation formula proposed by Batchelor, showed that the latter gives surprisingly precise results. The modification of the same method to obtain analytical corrections to the scaling law, taking into account the possible corrections induced by intermittency, is also proposed.
\end{abstract}

\section{Introduction}

The scaling of structure functions is a basic problem in the study of turbulence. The second and third order longitudinal structure function of the velocity $\boldsymbol{u}$ are respectively defined as

$$
\begin{aligned}
D_{l l}(r) & =\left\langle\left(u\left(x_{1}+r\right)-u\left(x_{1}\right)\right)^{2}\right\rangle \\
D_{l l l}(r) & =\left\langle\left(u\left(x_{1}+r\right)-u\left(x_{1}\right)\right)^{3}\right\rangle
\end{aligned}
$$

with $u$ the velocity component in the $x_{1}$-direction and $\langle\cdot\rangle$ denoting an ensemble average. In the inertial range of high Reynolds number turbulence, these structure functions were proposed to scale as $[1,2]$ :

$$
\begin{gathered}
D_{l l}(r) \sim(\varepsilon r)^{2 / 3} \\
D_{l l l}(r) \sim \varepsilon r,
\end{gathered}
$$

with the dissipation defined as

$$
\varepsilon=\nu\left\langle\frac{\partial u_{i}}{\partial x_{j}} \frac{\partial u_{i}}{\partial x_{j}}\right\rangle
$$

Email address: le.fang@ec-lyon.fr (Le Fang).

Preprint submitted to Elsevier Science

June 23, 2009 


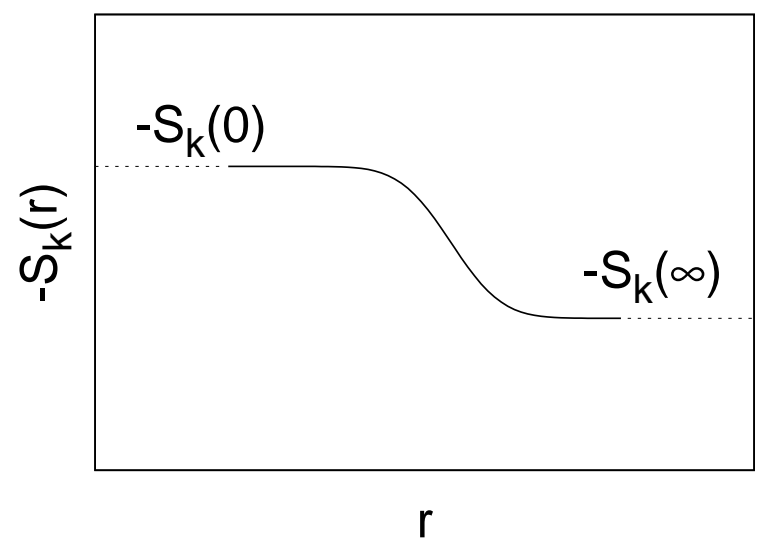

Figure 1. Schematic of the velocity increment skewness in isotropic turbulence. Two plateaus are expected at very large Reynolds number: one for $r \ll \eta$ the other for $\eta \ll r \ll L$

and $\nu$ the kinematic viscosity. Later, Kolmogorov [3] proposed a correction to the scaling of $D_{l l}(r)$, taking into account the increasingly intermittent character of the energy flux $\varepsilon_{f}(r)$ when the scale $r$ is decreased. This correction is however small and disputed. For example, several authors argued that the anomalous scaling (as observed experimentally by e.g. [4]) may stem from a finite Reynolds number effect [5,6], and it was proposed that the $2 / 3$ law may be satisfied when the Reynolds number tends to infinity. The second relation is an exact relation, which can be derived from the Navier-Stokes equations, be it only in the limit of very large Reynolds number. Indeed, for stationary isotropic incompressible turbulence one can derive

$$
D_{l l l}(r)=6 \nu \frac{d D_{l l}(r)}{d r}-\frac{4}{5} \varepsilon r .
$$

For scales much larger than the viscous scale, the first term on the right hand side vanishes so that (4) is obtained. Equation (6) relates the second and third order structure functions. So if $D_{l l l}(r)$ or the skewness

$$
S_{k}(r)=D_{l l l}(r) /\left(D_{l l}(r)\right)^{3 / 2}
$$

is known, the second order structure function can be computed using (6). A first attempt is substituting a constant skewness, as was proposed by Obukhov [7] (see also [8], chapter 22, or [9]). Indeed, in the inertial range, from (3) and (4) it is clear that the skewness of the longitudinal velocity increment should be a constant (in the absence of intermittency corrections). For $r \ll \eta$, with $\eta$ the viscous cut-off scale, the flow can be considered as smooth, so that the velocity increments scale linearly with the separation distance. This gives the trivial scaling

$$
\begin{aligned}
D_{l l}(r) & \sim r^{2} \\
D_{l l l}(r) & \sim r^{3}
\end{aligned}
$$

so that also at small scales the skewness should be a constant. Around $\eta$, the skewness is however not constant, since the plateaus at small and large $r$ do not have the same value, as sketched in Figure 1. Let us call these two values $S_{k}(0)$ and $S_{k}(\infty)$. These two values are relatively close, differing by approximately a factor two. Note that intermittency corrections change this discussion slightly, both at small and large, scales, but we will postpone this discussion to section 4 . 
In order to well represent the transition of the scaling exponent from 2 to $2 / 3$, Batchelor proposed an interpolation formula for the structure function [10], and this result has been widely used and adapted [11-13]:

$$
\frac{D_{l l}(r)}{v^{2}}=\frac{2(r / \eta)^{2 / 3}}{\left[1+(C \eta / r)^{2}\right]^{2 / 3}},
$$

with $v=(\nu \varepsilon)^{1 / 4}$ the Kolmogorov velocity, $\eta=\nu^{3 / 4} \varepsilon^{-1 / 4}$ is the Kolmogorov scale, and $C$ is a constant. This form was proposed because of its simplicity, not because of any underlying physics. It is in reasonable agreement with experiments.

In the present communication we present results on the transition region of the second-order structure function between the inertial range and the viscous range. The method is based on an extension of the work by Obukhov. Indeed, we use the assumption of a constant skewness for for $r \ll \eta$ and $\eta \ll r \ll L$ as a zeroth order solution. Then, starting from the inertial range we apply a perturbation method to evaluate the influence of the viscous damping. In the viscous range, we use Taylor-expansions to obtain an approximation of the influence of the non-constant skewness. Analytical expressions for the scaling exponent in both ranges are obtained. Results are compared to the Batchelor formula and a possible modification taking into account anomalous scaling is proposed.

\section{Analytical expressions for the scaling of $D_{l l}(r)$}

In homogeneous isotropic turbulence, The Kolmorogov equation (6), can be written as a function of $D_{l l}(r)$ and the skewness:

$$
S_{k}(r) D_{l l}(r)^{3 / 2}=6 \nu \frac{d D_{l l}(r)}{d r}-\frac{4}{5} \varepsilon r .
$$

We will in this section consider the two regions in which the skewness is constant at high Reynolds numbers, and compute analytically the corrections to the scaling of $D_{l l}(r)$, when $r$ approaches $\eta$.

\subsection{Corrections to the scaling in the dissipative range}

In the dissipative range, we define the following normalized quantities $r_{\eta}=r / \eta, D_{\eta}\left(r_{\eta}\right)=D_{l l}\left(\eta r_{\eta}\right) / v^{2}, D_{\eta}^{\prime}\left(r_{\eta}\right)=$ $d D_{\eta}\left(r_{\eta}\right) / d r_{\eta}$. Using Eq. (11), this leads to

$$
6 D_{\eta}^{\prime}-\frac{4}{5} r_{\eta}-S_{k}\left(r_{\eta}\right) D_{\eta}^{3 / 2}=0
$$

Suppose $S_{k}\left(r_{\eta}\right)=S_{k}(0)$ in the dissipative range, and let $w=D_{\eta}^{1 / 2}$. Eq. (12) can then be rewritten as

$$
12 w w^{\prime}-\frac{4}{5} r_{\eta}-S_{k}(0) w^{3}=0 .
$$

When $r_{\eta}$ tends to zero, from Taylor expansions we have

$$
w=\sum_{i=0}^{\infty} a_{i} r_{\eta}^{i}
$$

in which $a_{i}$ are unknown coefficients. Then the terms in Eq. (13) can be expressed:

$$
\begin{gathered}
w w^{\prime}=a_{1}^{2} r_{\eta}+3 a_{1} a_{2} r_{\eta}^{2}+\left(4 a_{1} a_{3}+2 a_{2}^{2}\right) r_{\eta}^{3}+\left(5 a_{1} a_{4}+5 a_{2} a_{3}\right) r_{\eta}^{4}+\ldots \\
w^{3}=a_{1}^{3} r_{\eta}^{3}+3 a_{1}^{2} a_{2} r_{\eta}^{4}+\left(3 a_{1} a_{2}^{2}+3 a_{1}^{2} a_{3}\right) r_{\eta}^{5}+\ldots
\end{gathered}
$$


Substituting Eqs. (15) and (16) into (13), and comparing the same order terms of $r_{\eta}$, we finally have

$$
w=\frac{1}{\sqrt{15}} r_{\eta}+\frac{S_{k}(0)}{720} r_{\eta}^{3}+\frac{\sqrt{15} S_{k}(0)^{2}}{345600} r_{\eta}^{5}+\frac{S_{k}(0)^{3}}{11059200} r_{\eta}^{7}+\frac{133 S_{k}(0)^{4}}{47775744000 \sqrt{15}} r_{\eta}^{9} \ldots
$$

and the structure function is

$$
D_{\eta}\left(r_{\eta}\right)=\frac{1}{15} r_{\eta}^{2}+\frac{S_{k}(0)}{360 \sqrt{15}} r_{\eta}^{4}+\frac{S_{k}(0)^{2}}{129600} r_{\eta}^{6}+\frac{S_{k}(0)^{3}}{3317760 \sqrt{15}} r_{\eta}^{8}+\frac{67 S_{k}(0)^{4}}{89579520000} r_{\eta}^{10} \cdots
$$

The local scaling exponent $n$ is defined as

$$
n=\frac{d \log D_{\eta}}{d \log r_{\eta}}=\frac{d D_{\eta}}{d r_{\eta}} \frac{r_{\eta}}{D_{\eta}}
$$

We compute its value from (18) to obtain, with $4^{\text {th }}$ order precision:

$$
n \approx 2\left(\frac{\frac{1}{15}+\frac{S_{k}(0)}{180 \sqrt{15}} r_{\eta}^{2}+\frac{S_{k}(0)^{2}}{43200} r_{\eta}^{4}+\frac{S_{k}(0)^{3}}{829440 \sqrt{15}} r_{\eta}^{6}+\frac{67 S_{k}(0)^{4}}{17915904000} r_{\eta}^{8}}{\frac{1}{15}+\frac{S_{k}(0)}{360 \sqrt{15}} r_{\eta}^{2}+\frac{S_{k}(0)^{2}}{129600} r_{\eta}^{4}+\frac{S_{k}(0)^{3}}{3317760 \sqrt{15}} r_{\eta}^{6}+\frac{67 S_{k}(0)^{4}}{89579520000} r_{\eta}^{8}}\right)
$$

Note that the corrections to the value 2 are a function of the longitudinal velocity derivative skewness $S_{k}(0)$.

\subsection{Viscous corrections to the inertial range}

From Eq. (11), using the assumption of a constant skewness, one can also obtain

$$
\frac{d S_{k}(r)}{d r}=\frac{d}{d r}\left(D_{l l}(r)^{-3 / 2}\left(6 \nu \frac{d D_{l l}(r)}{d r}-\frac{4}{5} \varepsilon r\right)\right)=0 .
$$

Introducing the Taylor micro-scale $\lambda=u_{0} \sqrt{15 \nu / \varepsilon}$, where $u_{0}=\sqrt{2 k / 3}$ is the characteristic velocity, and $r_{\lambda}=r / \lambda, D_{\lambda}\left(r_{\lambda}\right)=\frac{D_{l l}\left(\lambda r_{\lambda}\right)}{u_{0}^{2}}, D_{\lambda}^{\prime}\left(r_{\lambda}\right)=\frac{d D_{\lambda}\left(r_{\lambda}\right)}{d r_{\lambda}}$, the equation can be written as

$$
30\left(\frac{\eta}{\lambda}\right)^{2} D_{\lambda} D_{\lambda}^{\prime \prime}-45\left(\frac{\eta}{\lambda}\right)^{2} D_{\lambda}^{\prime 2}+6 r_{\lambda} D_{\lambda}^{\prime}-4 D_{\lambda}=0 .
$$

Considering the Reynolds number

$$
\operatorname{Re}_{\lambda}=\frac{u_{0} \lambda}{\nu}=\frac{1}{\sqrt{15}}\left(\frac{\lambda}{\eta}\right)^{2}
$$

we can rewrite Eq. (22) as

$$
30 \frac{\sqrt{15}}{\operatorname{Re}_{\lambda}} D_{\lambda} D_{\lambda}^{\prime \prime}-45 \frac{\sqrt{15}}{\operatorname{Re}_{\lambda}} D_{\lambda}^{\prime 2}+6 r_{\lambda} D_{\lambda}^{\prime}-4 D_{\lambda}=0 .
$$

When the Reynolds number is large, i.e. $\delta=\sqrt{15} / \operatorname{Re}_{\lambda} \ll 1$, the perturbation method can be applied. In this case the differential equation (24) is rewritten as

$$
30 \delta D_{\lambda} D_{\lambda}^{\prime \prime}-45 \delta D_{\lambda}^{\prime 2}+6 r_{\lambda} D_{\lambda}^{\prime}-4 D_{\lambda}=0,
$$

and the general solution can be expanded using $\delta$ :

$$
D_{\lambda}=\sum_{n=0}^{\infty} \delta^{n} D_{\lambda n} .
$$


Substituting (26) into (25), the $0^{\text {th }}$ order equation for $\delta$ is

$$
6 r_{\lambda} D_{\lambda 0}^{\prime}-4 D_{\lambda 0}=0 .
$$

The general solution which is found is

$$
D_{\lambda 0}\left(r_{\lambda}\right)=A_{0} r_{\lambda}^{2 / 3} .
$$

The coefficient $A_{0}$ can be determined by using the skewness value when $r_{\lambda} \rightarrow \infty$. We easily obtain

$$
A_{0}=15^{1 / 6} \operatorname{Re}_{\lambda}^{1 / 3} C_{0},
$$

in which

$$
C_{0}=\left(\frac{-4}{5 S_{k}(\infty)}\right)^{2 / 3}
$$

is a constant which does not depend on the Reynolds number. Note that the value of skewness $S_{k}(\infty)$ is usually not the same as $S_{k}(0)$.

Similarly, we can write the $1^{\text {st }}$ order equation for $\delta$ :

$$
30 D_{\lambda 0} D_{\lambda 0}^{\prime \prime}-45 D_{\lambda 0}^{\prime 2}+6 r_{\lambda} D_{\lambda 1}^{\prime}-4 D_{\lambda 1}=0 .
$$

From the solution of $D_{\lambda 0}$, i.e. Eq. (28), the solution of $D_{\lambda 1}$ can be obtained:

$$
D_{\lambda 1}\left(r_{\lambda}\right)=-\frac{10 A_{0}^{2}}{3 r_{\lambda}^{2 / 3}}+A_{1} r_{\lambda}^{2 / 3} .
$$

Since $D_{\lambda 1}$ should tend to 0 when $x \rightarrow \infty$, we have the coefficient $A_{1}=0$, thus

$$
D_{\lambda 1}(x)=-\frac{10 A_{0}^{2}}{3 r_{\lambda}^{2 / 3}} .
$$

Note that when $x \rightarrow 0$, this $1^{\text {st }}$ order solution can not been applied. It means that the perturbative solution is not correct when the two-point distance $r$ is very small, i.e. in the dissipative range.

Therefore, the general solution with $1^{\text {st }}$ order precision can be written as

$$
D_{\lambda}\left(r_{\lambda}\right)=A_{0} r_{\lambda}^{2 / 3}-\frac{10}{3} A_{0}^{2} r_{\lambda}^{-2 / 3} .
$$

It might seem strange at first sight that the constant $A_{0}$ depends on the Reynolds number. This comes from the fact that the increment distance is normalized by the Taylor scale, which is also a function of the Reynolds number. This dependence vanishes if we write the expression as a function of $r_{\eta}$ :

$$
D_{\eta}\left(r_{\eta}\right)=C_{0} r_{\eta}^{2 / 3}-\frac{10}{3} C_{0}^{2} r_{\eta}^{-2 / 3} .
$$

Higher order solutions can also been solved using the same method. For instance the solution with $4^{\text {th }}$ order precision is

$$
D_{\eta}\left(r_{\eta}\right)=C_{0} r_{\eta}^{2 / 3}-\frac{10}{3} C_{0}^{2} r_{\eta}^{-2 / 3}-\frac{125}{9} C_{0}^{3} r_{\eta}^{-2}-\frac{4750}{27} C_{0}^{4} r_{\eta}^{-10 / 3}-\frac{290000}{81} C_{0}^{5} r_{\eta}^{-14 / 3} .
$$

The scaling exponent with $4^{\text {th }}$ order precision is

$$
n=\frac{2}{3}\left(\frac{1+\frac{10}{3} C_{0} r_{\eta}^{-4 / 3}+\frac{125}{3} C_{0}^{2} r_{\eta}^{-8 / 3}+\frac{23750}{27} C_{0}^{3} r_{\eta}^{-12 / 3}+\frac{2030000}{81} C_{0}^{4} r_{\eta}^{-16 / 3}}{1-\frac{10}{3} C_{0} r_{\eta}^{-4 / 3}-\frac{125}{9} C_{0}^{2} r_{\eta}^{-8 / 3}-\frac{4750}{27} C_{0}^{3} r_{\eta}^{-12 / 3}-\frac{290000}{81} C_{0}^{4} r_{\eta}^{-16 / 3}}\right)
$$

The scaling exponent is always larger than $2 / 3$, and tends to $2 / 3$ when $x \rightarrow \infty$. It is in agreement with the arguments by Barenblatt [14], who argues that the anomalous scaling with exponents slightly 

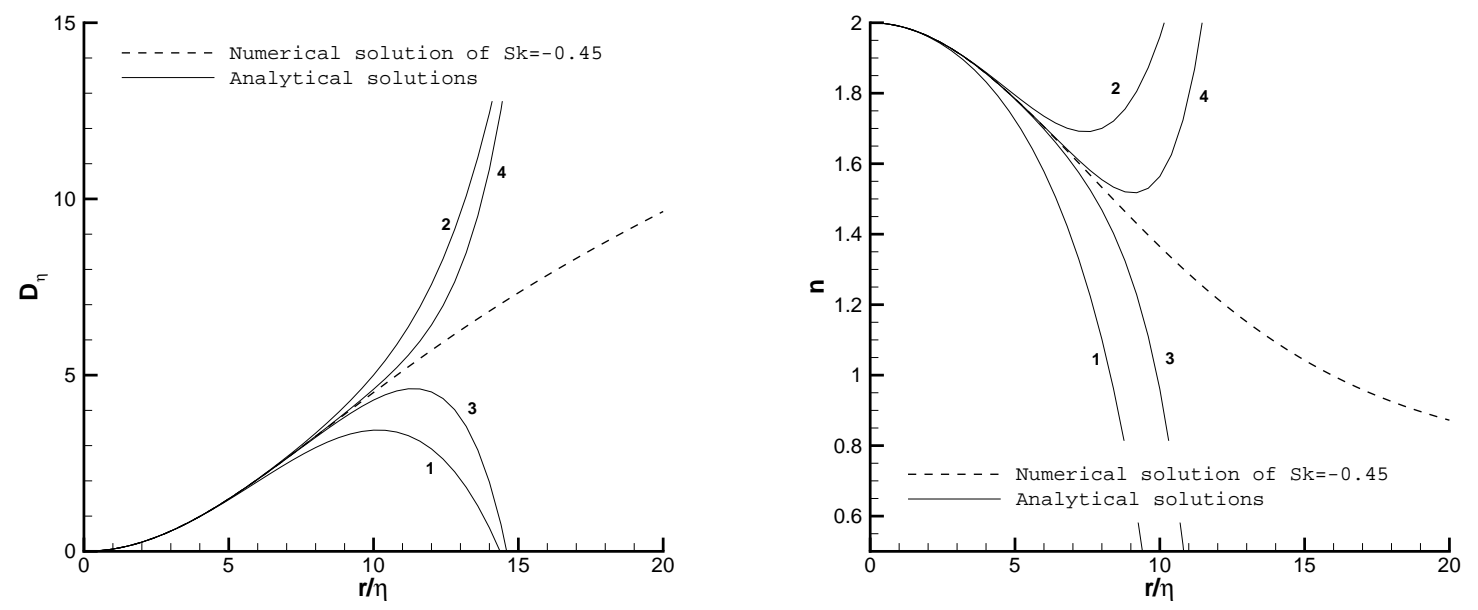

Figure 2. Comparison between the numerical solution and the analytical solutions in the dissipative range. In each figure, the perturbative solutions have $1^{\text {st }}$ to $4^{t h}$ order precisions, the numbers in the figure denote the orders respectively. (a) Structure function. (b) Scaling exponents

larger than $2 / 3$ observed in several works, is an artefact simply due to the effect of viscosity. However in Barenblatt's paper, only a general expression is derived by using dimensional analysis, in which the coefficients are not determined. In the present work we obtain a quantitative correction stemming from the effect of molecular viscosity.

\section{Comparison of the corrections with existing results}

\subsection{Comparison to the approximation of a constant skewness}

In order to verify the analytical corrections, we compare them with the exact numerical solution of the original differential equation (12), i.e. the approximation of a constant skewness.

The comparison in the dissipative range is shown in Fig. (2). The value of the skewness is fixed at $S_{k}(0)=-0.45$. Both structure functions and local scaling exponents are shown for different orders of the expansion. When $r$ is small, the analytical solutions are in good agreement with the numerical solution. The $1^{\text {st }}$ order solution is satisfied in the range about $0<r_{\eta}<6$, and for the $4^{\text {th }}$ order solution it is about $0<r_{\eta}<10$.

Figure 3 shows comparisons between the numerical solution and the perturbative solutions when $r \gg$ $\eta$. The skewness value is fixed as $S_{k}(\infty)=-0.2$. The perturbative solutions are close to the numerical solution of the constant skewness equation. The solution of $1^{s t}$ order precision coincides when $r / \eta>60$, and the solution of $4^{t h}$ order precision is in good agreement when $r / \eta>25$.

Note that the parameters can change with different values of the skewness. The skewness value in the dissipative range $S_{k}(0)$ can be obtained from spectral theory such as the direct interaction approximation [15] or experimental results [16], but there is no analytical result for the transition between dissipative range and inertial range. We mention however the work by Tatarskii, who suggested an ad hoc interpolation formula between the value of the longitudinal velocity derivative skewness and the skewness 

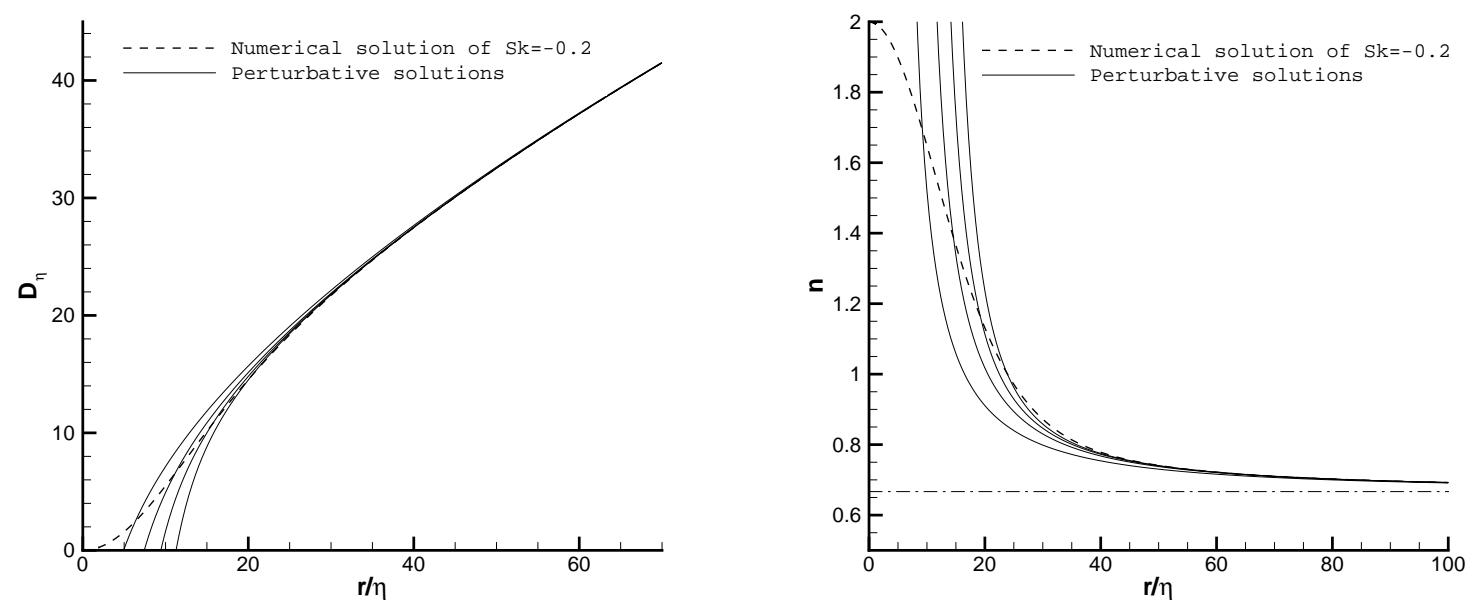

Figure 3. Comparisons between the numerical solution and the perturbative solutions when $r \gg \eta$. In each figure, the perturbative solutions have $1^{\text {st }}$ to $4^{\text {th }}$ order precisions, from left to right respectively. (a) Structure functions. (b) Scaling exponents. The horizontal line is the constant value $2 / 3$.

in the inertial range [9].

\subsection{Comparison with Batchelor's formula}

When $r_{\eta}$ is small, Batchelor's formula (8) can be expanded using Taylor series:

$$
D_{\eta}=2 C^{-4 / 3} r_{\eta}^{2}-\frac{4}{3} C^{-4 / 3} r_{\eta}^{4}+\frac{10}{9} C^{-16 / 3} r_{\eta}^{6}-\frac{80}{81} C^{22 / 3} r_{\eta}^{8}+\ldots
$$

Comparing Eq. (38) with (18), the $r^{2}$ terms yields $2 C^{-4 / 3}=1 / 15$, i.e. $C=30^{3 / 4} \approx 13$. $C$ is therefore not a free constant. This formula with constant value $C=13$ has been applied widely in many studies e.g. $[11,12]$. Indeed this constant value satisfies well the behavior of $D_{\eta}$ in the dissipation range. Here we will focus on the performance of Batchelor's formula to estimate the scaling exponent $n$.

Comparing Eq. (38) with the analytical solution of scaling exponent (20), and ignoring the high-order terms, we can obtain:

$$
\frac{6 C^{2}-8 r_{\eta}^{2}}{3 C^{2}-2 r_{\eta}^{2}}=\frac{48 \sqrt{15}+4 S_{k}(0) r_{\eta}^{2}}{24 \sqrt{15}+S_{k}(0) r_{\eta}^{2}}
$$

and the constant value $C$ can be solved to yield:

$$
C=4 \sqrt{-\frac{\sqrt{15}}{S_{k}(0)}} .
$$

So the correction to the scaling exponent, given by (20) is a function of the velocity derivative skewness, whereas the first order correction given by Batchelor's formula is a function of $C$. Taking the correct value $C=13$ corresponds to a velocity derivative skewness $S_{k}(0) \approx-0.38$. This is actually rather close to the value obtained in experiments, even though experimental scatter is large [17]. To illustrate, if we use $S_{k}(0)=-0.45$, and from Eq. (40) we obtain $C \approx 11.7$. 

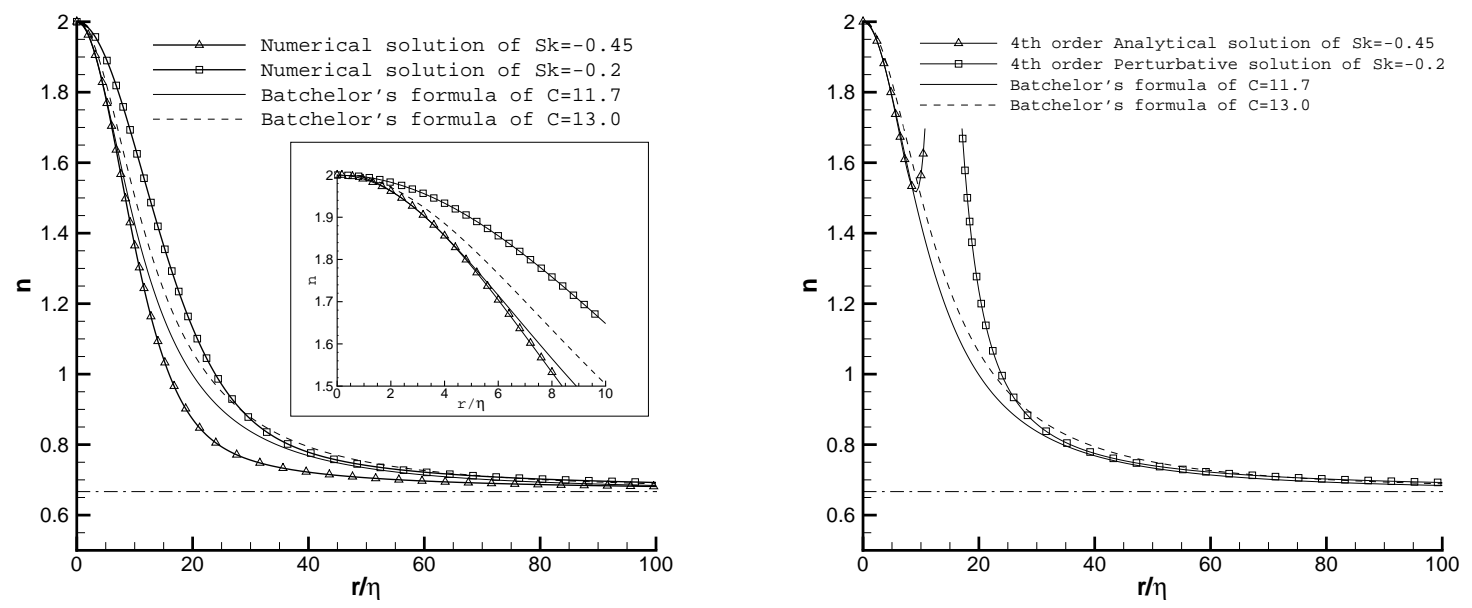

Figure 4. Comparisons between Batchelor's formula and: (a) numerical solutions of constant skewness. (b) analytical solutions of constant skewness. The horizontal line is constant $2 / 3$.

In Fig. 4, Batchelor's interpolation formula (8) is compared with the numerical and analytical solutions of constant skewness. In the dissipative range we fix $S_{k}=-0.45$ and in the inertial range $S_{k}=-0.2$. Both results of $C=13$ and $C=11.7$ are shown, and $C=11.7$ is in better agreement with the $S_{k}=-0.45$ solution in the dissipative range (see the enlarged subfigure). From Fig. 4 (a), we can conclude that Batchelor's formula denotes a transition between the solutions of different values of the skewness. The formula with $C=11.7$ is in good agreement with the $S_{k}=-0.45$ solution in the dissipative range, and also in agreement with the $S_{k}=-0.2$ solution in the range $r / \eta>30$, i.e. the inertial range. Also our analytical expression are in good agreement with the constant skewness assumption in these two ranges respectively, as shown in Fig. 4 (b).

The comparison of the structure functions given by Batchelor's formula and the analytical expressions of constant skewness are shown in Fig. 5. There is a small difference at the small scales, since we changed the value of $C$ and the relation $D_{\eta} \approx r_{\eta}^{2} / 15$ is no longer satisfied. It means that Batchelor's formula does not agree simultaneously with the analytical expression obtained for $D_{l l}(r)$ using the constant skewness assumption and with the corrections to the scaling exponent given by the same approach, unless $S_{k}(0)=$ 0.38. We admit however that the difference is small, and that Batchelor's formula does a surprisingly good job. If an explicit expression for the scaling exponent is needed our expression (40) can supply this as a function of $S_{k}(0)$.

In Fig. 6 it is observed how Batchelor's formula performs in the beginning of the inertial range. For small values of $r / \eta$ the scaling exponent is close to the prediction given by the assumption that $S_{k}=-0.2$. For larger values it approaches the solution for $S_{k}=-0.45$. So Batchelor's formula for the scaling exponent corresponds to the case in which the skewness transitions between two values around $r / \eta \approx 100$. 


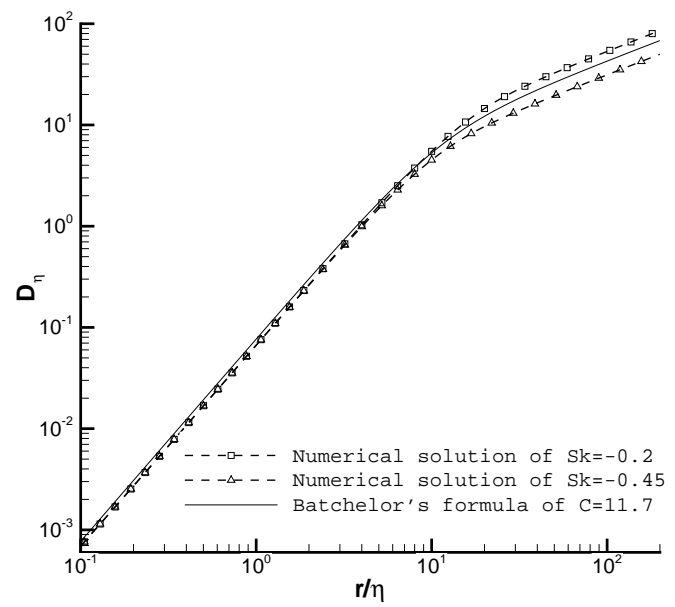

Figure 5. Comparisons of the structure functions, between Batchelor's formula the numerical solutions of constant skewness.

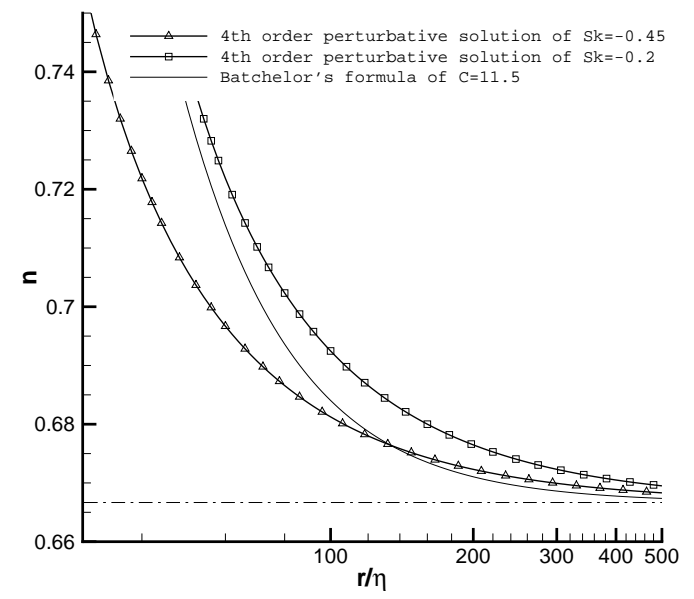

Figure 6. Comparisons between Batchelor's formula the analytical solutions of constant skewness, in inertial range. The horizontal line is the constant value $2 / 3$.

\section{Modifications to take into account anomalous scaling}

As stated in the introduction, intermittency corrections to the scaling of $D_{l l}(r)$ might exist. We do not want to argue in favour or against these corrections, which is an issue beyond the scope of the present paper. We will just outline how the above results change if $D_{l l}(r)$ scales as

$$
D_{l l}(r) \sim r^{\zeta_{2}}
$$

with $\zeta_{2} \neq 2 / 3$. If we replace the assumption $S k(r)=D_{l l l} / D_{l l}^{3 / 2}=$ constant by the assumption 


$$
S k^{*}(r)=D_{l l l} / D_{l l}^{1 / \zeta(2)}=\text { constant, }
$$

the proposed perturbation method can now equally well be applied to equation (6), changing only the numerical values.

\section{Conclusion}

In the present work the approach of Obukhov assuming a constant skewness was used to obtain analytical corrections to the scaling of the second order structure function, starting from Kolmogorov's $4 / 5$ law. These corrections can be used in model applications in which explicit expressions, rather than numerical solutions are needed.

In the far dissipation range and in the inertial range, these expressions should give a relatively adequate representation of the structure function and the scaling exponent. The comparison with an interpolation formula proposed by Batchelor, showed that the latter gives surprisingly precise results. It is shown that Batchelor's formula in the dissipation range coincides to first order, with the assumption of a constant skewness, with the particular value $S_{k}(0)=0.38$. In the beginning of the inertial range, the scaling exponent does not coincide with the assumption of a constant skewness.

The modification of the same method to obtain analytical corrections to the scaling law, taking into account the possible corrections induced by intermittency, was also proposed.

\section{References}

[1] A. N. Kolmogorov. The local structure of turbulence in incompressible viscous fluid for very large reynolds numbers. Dokl. Akad. Nauk SSSR, 30:301, 1941.

[2] A.N. Kolmogorov. Dissipation of energy in locally isotropic turbulence. Dokl. Akad. Nauk SSSR, 32:19, 1941.

[3] A.N. Kolmogorov. A refinement of previous hypotheses concerning the local structure of turbulence in a viscous incompressible fluid at high reynolds number. J. Fluid Mech., 13:82, 1962.

[4] F. Anselmet, Y. Gagne, E.J. Hopfinger, and R.A. Antonia. High-order velocity structure functions in turbulent shear flows. Journal of Fluid Mechanics, 140:63, 1984.

[5] J. Qian. Normal and anomalous scaling of turbulence. Physical Review E, 58(6):7325-7329, 1998.

[6] V.S. L'vov and I. Procaccia. intermittencyj in hydrodynamic turbulence as intermediate asymptotics to kolmogorov scaling. Phys. Rev. Lett., 74:2690, 1995.

[7] A. M. Obukhov. The local structure of atmospheric turbulence. Dokl. Akad. Nauk. SSSR, 67:643, 1949.

[8] A. S. Monin and A. M. Yaglom. Statistical Fluid Mechanics. Mechanics of Turbulence, Vol 2. MIT Press, 1975.

[9] V. I. Tatarskii. Use of the $4 / 5$ kolmogorov equation for describing some characteristics of fully developed turbulence. Physics of Fluids, 17:035110, 2005.

[10] G. K. Batchelor. Pressure fluctuations in isotropic turbulence. Proc. Cambridge Philos. Soc., 47:359, 1951.

[11] D. Lohse and A. Muller-Groeling. Bottleneck effects in turbulence: scaling phenomena in r versus p space. Physical Review Letters, 74(10):1747, 1995.

[12] C. Meneveau. Transition between viscous and inertial-range scaling of turbulence structure functions. Physical Review E, 54(4):3657, 1996.

[13] L. Chevillard, B. Castaing, E. Lvque, and A. Arneodo. Unified multifractal description of velocity increments statistics in turbulence: Intermittency and skewness. Phys. D, 218:77, 2006.

[14] G.I. Barenblatt, A.J. Chorin, and V.M. Prostokishin. Comment on the paper on the scaling of three-dimensional homogeneous and isotropic turbulence by benzi et al. Physica D, 127:105-110, 1999. 
[15] R.H. Kraichnan. The structure of isotropic turbulence at very high Reynolds numbers. J. Fluid Mech., 5:497-543, 1959.

[16] G. K. Batchelor. The theory of homogeneous turbulence. Cambridge University Press, 1953.

[17] C. W. Van Atta and R. A. Antonia. Reynolds number dependence of skewness and flatness factors of turbulent velocity derivatives. Physics of Fluids, 23(2):252-257, 1980. 